\title{
A Reliable Cross Layer Routing Scheme (CL-RS) for Wireless Sensor Networks to Prolong Network Lifetime
}

\author{
Kusumamba $S$ \\ Dept. of PG Studies in Computer Science and Applications \\ Bangalore University \\ Bangalore, India \\ E-mail: kusuma123.sreeram@gmail.com
}

\author{
S M Dilip Kumar \\ Dept. of Computer Science and Engineering \\ University Visvesvaraya College of Engineering \\ Bangalore, India \\ E-mail: dilipkumarsm.uvce@bub.ernet.in
}

\begin{abstract}
Design of conventional protocols for wireless sensor networks(WSN) are mainly based on energy management. The solutions for layered protocol of the WSN network are inefficient as sensors network mainly delivers real-time content thus, cross layer communication between layers of the protocol stack is highly required. In this paper, a reliable cross layer routing scheme $(C L-R S)$ is proposed to balance energy to achieve prolonged lifetime through controlled utilization of limited energy. $C L-R S$ considers 2 adjacent layers namely, MAC layer and network layer. Optimization issues are identified in these two layers and solutions are provided to reduce energy consumption thereby increasing network lifetime. To achieve higher energy efficiency MAC layer protocols compromise on packet latency. It is essential to attempt reduce the end-to-end delay and energy consumption using low duty cycle cross layer MAC $(C L-M A C)$. The joint optimization design is formulated as a linear programming problem. The network is partitioned into four request zones to enable increase in network performance by using an appropriate duty cycle and routing scheme. We demonstrate by simulations that the strategy designed by combining $(C L-R S)$ and $(C L-M A C)$ algorithms at each layer significantly increases the network lifetime and a relation exists between the network lifetime maximization and the reliability constraint. We evaluate the performance of the proposed scheme under different scenarios using ns-2. Experimental results shows that proposed scheme outperforms the layered AODV in terms of packet loss ratio, end-to-end delay, control overhead and energy consumption.
\end{abstract}

Keywords-Cross layer, Optimization, Reliability, Routing, Network lifetime, Wireless sensor network.

\section{INTRODUCTION}

Lifetime maximization in non-rechargeable powered batteries in WSN has been extensively studied in recent years. There is a increasing body of literature survey on energy conservation in WSN. Since energy is a scarce resource in WSN, all layers of the protocol stack has contributed to energy conservation, designing energy-aware algorithms across different layers in order to prolong lifetime of WSN is necessary [1]. The conventional layered protocols are individually developed and optimized for achieving high performance in the network. However, joint optimization of the protocols in each layer are not considered to improve the overall network performance while reducing the energy consumption. Considering the energy and resource constraint of WSN, crosslayer design exploit dependencies and interaction across layers into a unified communication framework [2], [3]. Cross layer design is currently one of the most active research areas in WSN. Cross layer interaction allows communication between possible adjacent layers of protocol stack. Cross layer was thought of to address problem of energy consumption, reliability, wireless links, packet loss and delay problems observed in the network. Cross layer approach involves different layers for information sharing where, these information can be used as a input for algorithms in the protocol design of WSN [4]. In this paper, we propose cross layer strategy that prolong the network lifetime under the reliability constraint. We focus on a sensor application, where a collection of distributed sensor nodes periodically report data to a sink node. In order to guarantee the success probability of data aggregation from sensor to sink, the proposed strategy optimizes the network layer and MAC layer. At the network layer, we design a new joint cross layer routing algorithm that is different from conventional energy-efficient routing algorithms [5], [6], [7]. At the MAC layer, we design energy efficient low duty cycle scheduling. Ideas in both the layers is formulated into a linear optimization problem. Hence, the objective is to create a flexible cross layer design for WSN that considers the criticality of the energy conservation thereby increasing network lifetime. The rest of the paper is organized as follows: Section II presents the brief review of related works on cross layer design in WSN. The network model and assumptions is presented in Section III. Problem statement and formulation are given in Section IV. The proposed cross layer routing scheme $C L-R S$ for routing is explained in Section V. In Setion VI, simulations and performance analysis are presented. In Section VII, the concluding remarks and future work is presented.

\section{RELATED WORKS}

In this section, we present a brief overview of some of the existing works on cross layer routing in WSN related to this work. In [8], a load balanced forwarding scheme is proposed in which sensor nodes will select the next alternative relay by considering buffer occupancy level of the neighbour at the time of delivery. The authors in [9] proposed a routing protocol by considering residual energy and link quality of network to determine the route to the destination, where it does not consider the load on the network which leads to congestion. A reliable energy-efficient multi-level routing algorithm is pro- 
TABLE I: Notations used

\begin{tabular}{|l|l|}
\hline$E_{\text {init }}$ & Initial energy of a node \\
$Q_{i}$ & Packet generation rate \\
$N_{i}$ & Set of neighbour nodes reached by node $i$ \\
$e_{i j}$ & Energy to transmit a packet from node $i$ to node \\
$q_{i j}$ & $j$ \\
$E_{A E C}$ & Rate of packet flow from node $i$ to node $j$ \\
$S$ & Energy consumption of node to relay a packet in time t \\
$D$ & Set of source nodes \\
$N$ & Destination node or sink \\
$P_{i j}$ & Set of sensor nodes \\
$E_{t x}$ & Path between node $i$ to node $j$ \\
$E_{r x}$ & Energy needed by node for transmitting a packet \\
$E_{p r o}$ & Energy needed by node for receiving a packet \\
$T$ & Energy needed for processing a packet \\
$T R F$ & Network lifetime \\
$E(i, p)$ & Target end-to-end reliability factor \\
$E T R$ & Energy consumed by node $i$ in path p \\
\hline
\end{tabular}

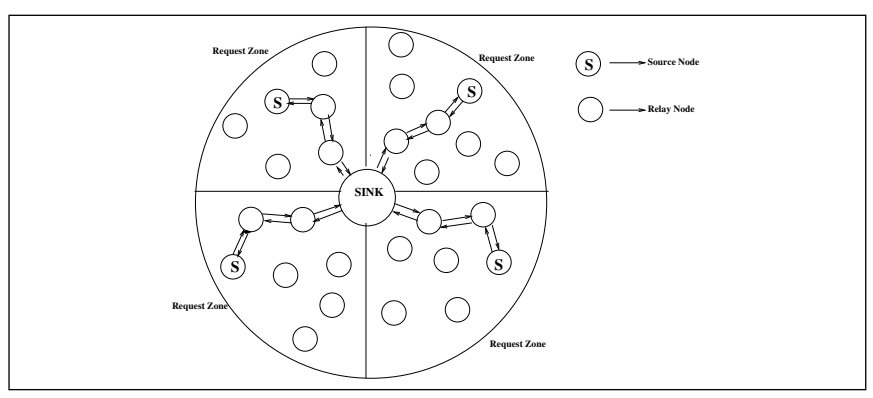

Fig. 1: Network Model

posed in [10] which considers residual energy, number of the neighbors and centrality of each node. This protocol reduces the energy consumption where it does not address problem of link quality. The data quality improvement using cross layer strategy is addressed in [11] considering privacy management policy coupled with a secure localization protocol. In [12], aIn this paper, Cross-Layer MAC protocol is propose to handle the latency of the packet unlike regular MAC layer this scheme proposes a multi flow data by considering routing layer buffer from neighbours, where it does not consider the end-to-end success transmission is proposed. Delay and energy aware cooperative medium access control (DEC_MAC) is proposed in [13] to balance the energy consumption of the sensor nodes by taking into account a node's residual energy a part of the relay selections metric to increase the network lifetime the selection metric ignores the quality of channel which is most important to minimize the energy in the network. A cross layer sleep scheduling design in service-oriented WSN aims to prolong lifetime while satisfying the service availability requirement at application layer is proposed in [14]. It is observed that the design does not contribute to network layer, in which energy is consumed high. The above stated protocols address the problem of increasing network lifetime to a major extent, however they cannot effectively overcome the reliability constraint with low end to end delay. In this paper, we propose a $C L-R S$ which can effectively increase the lifetime with minimum energy consumption with end-to-end reliability.

\section{NETWORK MODEL AND ASSUMPTIONS}

Network considered consists of one sink node and several sensor node.The sensor nodes are further divided into source nodes and relay nodes. Network is deployed in circular fashion and the sink is placed in the centre of the network as shown in Fig. 1 source nodes are placed on the circumference of the network. Periodically data is sent from source node to destination sink node using CBR traffic scheme. It is assumed that all the nodes have equal transmission range with an equal initial energy $E_{\text {init }}$. We also assume that all the packet size are of equal length and all the nodes have equal buffer queue size. The whole network is divided into four request zones in a quadrant fashion. Request zones are created by the sink node during the network deployment phase. Source node when it has data to send should establish a routing path only in its request zone. This avoids flooding of control packets and thus minimizing control overhead. The various notations used in this paper are stated in Table I. Network lifetime $(T)$ is the time from the time of the network deployment to the time first node expires. Network lifetime of a node $i$ is determined as follows:

$$
T=\frac{E_{\text {init }}(i)}{E_{A E C}(i)}
$$

where

$$
E_{A E C}=e_{i j} * q_{i j}
$$

and

$$
e_{i j}=E^{t x}+E^{r x}+E^{\text {pro }}
$$

Requisite energy needed for a node $i$ to receive and transmit packet to node $j$ is specified in Eq. 3 .

\section{PROBlem STATEMENT}

The problem is to compute a cross layer joint routing scheme to build an end-to-end routing path that maximizes the network lifetime with reliability constraint. Cross layer joint routing scheme is based on the node's local value of link quality, load on the node, residual energy and hop count. Cross layer MAC is developed as contribution to low duty cycle MAC. Maximising the network lifetime is completely depended on the residual energy and channel state information of each sensor node. Maximizing the network life time is the objective of finding energy efficient routes until first node expires. The cross-layer joint routing scheme is characterised and formulated as two linear programming problems as follows:

\section{A. Network Lifetime Maximisation problem}

Increasing the lifetime $T$ of a single node will contribute to increase in the lifetime of the network on whole. The objective function maximizes the network lifetime $T$ with subject to low energy consumption. The objective function is written as:

$$
\begin{aligned}
& \operatorname{maximize} T \\
& \text { subject to } \\
& q_{i j} \geq 0, \forall j \in N_{i}, \forall i \in N-D \\
& \sum_{j \in N_{i}} e_{i j} q_{i j} \leq E_{\text {init }}, \forall i \in N-D \\
& \sum_{j: i \in N_{j}} q_{i j}+T Q_{i}=\sum_{k \in N_{i}} q_{i k}, \forall i \in N-D
\end{aligned}
$$




\section{B. Energy Consumption Minimization problem}

We formulate a end-to-end routing path which minimizes the energy consumption in the network with reliability constrain. Minimizing the energy consumption of node $E_{A E C}$ to relay a packet in time $t$ is essential to increase the network lifetime. Reliability constraint is defined as the end-to-end reliable transmission ratio (RTR). End-to-end reliable transmission ratio can be achieved by hop to hop RTR. This can be formulated by linear minimization problem and the objective function is written as:

$$
\begin{array}{r}
\text { minimize } \sum_{i=1}^{n} E_{A E C}=\sum_{i=1}^{n} \sum_{i=1}^{p} q(i)+E(i, p) \\
\text { subject to } \prod_{i=1}^{n} R T R\left(S N R_{i j}, E_{i}\right) \geq T R F \\
R T R\left(S N R_{i j}, E_{i}\right)=1-\operatorname{ETR}\left(S N R_{i j}, E_{i}\right)
\end{array}
$$

Link quality is measured using link quality estimator (LQE) [9]. The LQE is measured in terms of signal-to-noise ratio (SNR). SNR of node $i$ to node $j$ is given by $S N R_{i j}$. The endto-end RTR is the product of hop-to-hop RTR and is computed by $\prod_{i=1}^{n} R T R$. RTR is the function of SNR.

\section{THE PROPOSED SCHEME}

For the proposed scheme, two different cross layer methods are utilized namely, cross layer routing scheme $(C L-R S)$ and cross layer MAC $(C L-M A C)$. For routing scheme, a new interface at network layer is designed such that an optimal route to the sink is chosen. In solving the routing problem using cross layer design, the goal is to choose the next relay node with an energy efficient channel with reliability constraint. Path selection is computed at the network layer and channel selection at the link layer. This forms the basis of cost functions that model the preferences of nodes in balancing energy in various layers. Cost functions quantify the performance benefits and energy costs associated with efficient relay node selection. The proposed joint routing scheme considers parameters of a node like residual energy, current queue length, hop count to sink and link quality. Reliability mainly depends upon considering LQE in the route selection process of the routing protocol. The LQE is measured using cross layer information of the network protocol stack. Every node is able to estimate the network conditions like load on the node, the end-to-end link quality, have information on the remaining energy of their neighbor nodes and number of hops to sink. To ensure reliability and to increase network lifetime every node dynamically plan the route discovery process as explained in Section V-A.

\section{A. Cross Layer Joint Routing Scheme $(C L-R S)$}

The core idea of the $C L-R S$ is to provide a framework where design issues like robust routing, congestion control and reliability are jointly considered. In $C L-R S$, a competition is triggered to select the best relay node. The competition is based on evaluation of the path cost function given in Eq. (8). The path cost function is calculated based on the residual energy, number of hops to the destination, traffic load of the node and link quality is given in Eq. (8). Source node after calculating the path cost function of all its neighbour nodes selects the best relay node. The proposed routing scheme is divided into two phases: the first phase is to check whether relay node will participate in the data transmission or not. The second phase is to choose a routing path such that Eq. (7) is minimized.

$$
A P C=\sum P C(i, j)
$$

where

$$
P C(i, j)=\alpha \frac{E_{\text {res }}}{E_{\max }}+\beta \frac{Q_{\text {cur }}}{Q_{\max }}+\gamma h o p(i, D)+L(i, j)
$$

The path cost function given in Eq. (8) considers residual energy, hop count, link quality and load on node. Eq. (4) and Eq. (5) are considered in formulating path cost function. By considering the load of the node, $C L-R S$ regulates congestion that incorporates a hop-to-hop congestion control by buffer occupancy analysis specified by current buffer queue length $\left(Q_{c u r}\right)$ to maximum queue length $\left(Q_{\max }\right)$. Link quality of a path is estimated hop-to-hop to increase the reliability. Link quality of node $i$ to node $j(L(i, j))$ is estimated using LQE. The residual energy of a node after time $t$ is given by $E_{\text {res }}$. Number of hops from node $i$ to the destination is given by hop $(i, D)$. These two phases are explained in the following:

1) Node's participation in communication: The basis of communication in $C L-R S$ is based on the initial assessment $(I A)$. To provide reliable and efficient end-to-end transmission service, $C L-R S$ will incorporate a communication functionality similar to AODV. The selection of the next-hop relay node is performed by means of contention of RREQ packets. For any node $i$ that wants to initiate a data transmission, broadcast a RREQ message to inform its neighbours that it has packets to send. Every neighbours of node $i$ upon receiving the RREQ packet decides whether to take part the communication process or not through an $I A$. The $I A$ is given by

$$
I A=\left\{\begin{array}{l}
L_{\text {rreq }}>l_{t h} \\
E_{\text {res }}>e_{t h} \\
Q_{\text {cur }}>q_{t h}
\end{array}\right.
$$

According to Eq. (9), it is necessary to choose only those nodes with higher $L Q E$ to participate in communication since they provide reliable links for data transmission. Therefore, it is necessary that $L Q E$ of HELLO message $\left(L_{r r e q}\right)$ should be above a threshold $\left(L_{t h}\right)$ for a node to participate. To maximise the node's life, $\left(E_{r e m}\right)$ should be above the threshold level $\left(E_{t h}\right)$. To prevent a node level congestion, $Q_{c u r}$ should be above the threshold $\left(Q_{t h}\right)$.

2) Route Discovery: Route discovery is done in the request zone of the source node. If the relay node has data to forward to the destination it should perform IA as stated in Eq. (9). If the relay node does not satisfy the $I A$, it drops the RREQ packet and backoff itself from the participation. It initiates a data transmission participation. If a node does not have a route previously available in its routing table, it broadcasts an RREQ to all its neighbours in its zone. If neighbour is not a destination, it rebroadcasts the RREQ packet. An intermediate node before rebroadcasting calculates the path cost according to Eq. (8) and add the value to accumulated path cost as given in Eq. (7) in the header of the RREQ. The intermediate node compares all RREQ packet with same destination and sequence ID, selects the lowest APC and then broadcast the minimum 
valued RREQ to all the neighbours in its zone as shown in Algorithm 1.In Algorithm 2, the destination node waits for all RREQ message until time $t$ after first RREQ has arrived. During time $t$, the destination node examines all the RREQ header and select minimum RREQ packet, unicasts a RREP packet back to the source node.
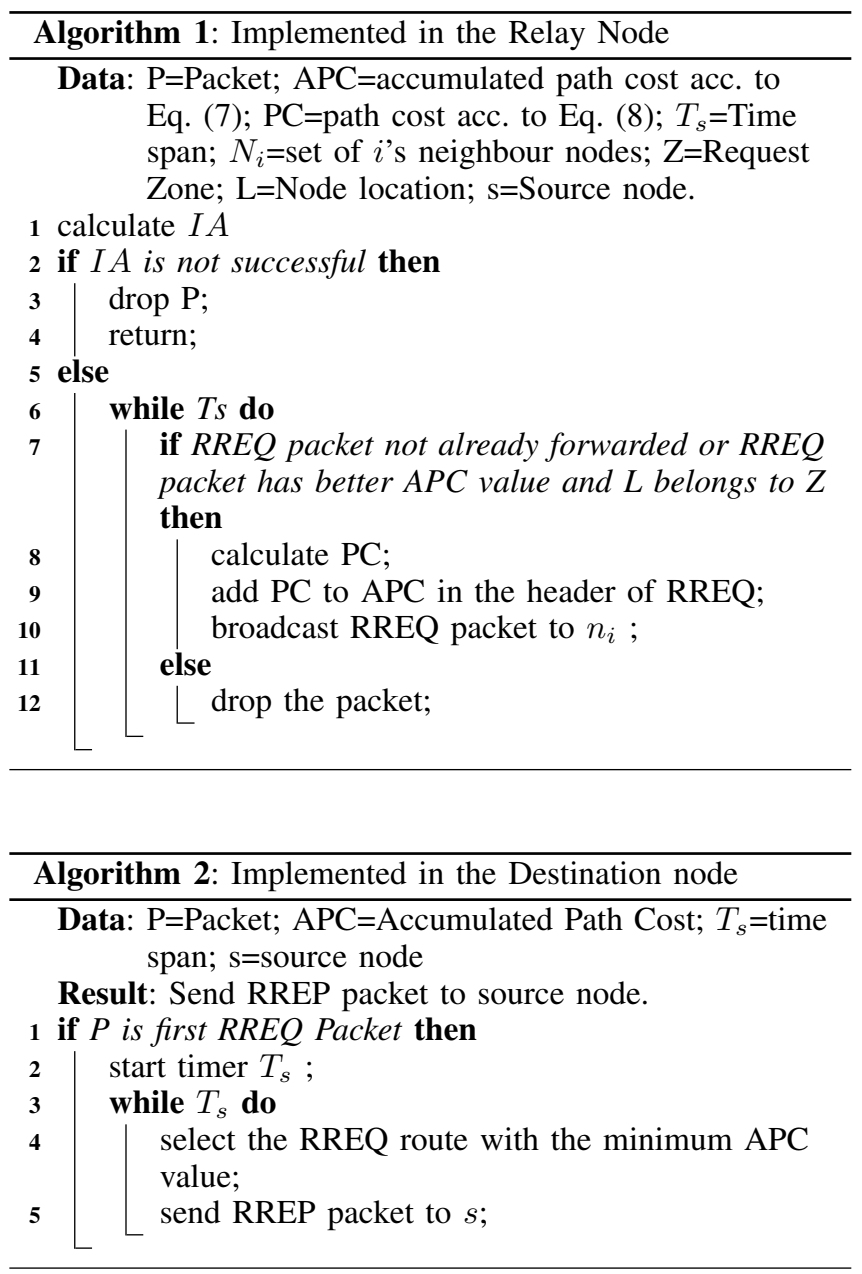

Route maintenance and recovery is important in WSN as sensors are exposed to external environment where exists noise interference and therefore the links are lost and it is a high overhead on the network to reestablish the lost link. $C L-R S$ uses local strategy to establish lost link by using SNR information provided by the MAC during the path cost calculation as stated in Eq. (8). When the SNR of a particular node is below the threshold level, the node alerts the neighbour about its status by which the whole network in that zone is aware of the weak links. $C L-R S$ fully utilizes the previous routing information stored in stale on-route node to reestablish the route path.

\section{B. Cross layer $M A C(C L-M A C)$}

In order to optimize the network performance in terms of energy consumption, adaptive sleep scheduling algorithms have been presented. The adaptive duty cycle utilize different metrics, such as network load, residual energy and link quality to adjust duty cycle of a node. The proposed $C L-M A C$ aims
TABLE II: Simulation Parameters List

\begin{tabular}{|c|l|}
\hline Parameter & Value \\
\hline Topology Size & $2000 \mathrm{~m} \times 2000 \mathrm{~m}$ \\
\hline Transmission range & $250 \mathrm{~m}$ \\
\hline Simulation Time & $5 \mathrm{M}$ \\
\hline Number of sinks & 1 \\
\hline Traffic Type & CBR \\
\hline MAC & MAC/802_11 \\
\hline Antina Model & Omni Antenna \\
\hline Transmitting power & 0.036 \\
\hline Receiving power & 0.024 \\
\hline Initial energy & 10 Joules \\
\hline Processing energy & 0.0022 \\
\hline
\end{tabular}

TABLE III: Simulation Parameters List

\begin{tabular}{|c|c|c|c|}
\hline Parameter & Section VI-A & Section VI-B & Section VI-C \\
\hline Number of nodes & 100 & 100 & $25-250$ \\
\hline Number of Connections & $10-60$ & 5 & 5 \\
\hline Packet generation rate & $1.50 \mathrm{pkts} / \mathrm{sec}$ & $0.25-.50 \mathrm{pkts} / \mathrm{sec}$ & $1.50 \mathrm{pkts} / \mathrm{sec}$ \\
\hline Load on node & $30 \mathrm{MB}$ & $30 \mathrm{MB}$ & $40 \mathrm{MB}$ \\
\hline Transfer rate & $200 \mathrm{kbps}$ & $200 \mathrm{kbps}$ & $200 \mathrm{kbps}$ \\
\hline
\end{tabular}

to reduce energy consumption and to increase lifetime of the network. When a source node establishes a routing path in its request zone using $C L-R S$, the $C L-M A C$ allows only nodes belonging to routing path to be awake while allowing other nodes in the zone to sleep mode. Thereby, energy is saved in all the nodes which are not part of the routing path during the communication time of source node and sink. The sink node after sending a RREP packet to source node also sends a broadcast message to all other nodes in the request zone that contains information about the nodes involved in routing path. The remaining nodes switch themselves into the sleep mode, thus saving energy.

\section{Vi. Simulation}

$n s-2$ [15] simulator is used to implement the proposed cross layer scheme. The performance of the proposed scheme and layered AODV is evaluated and compared. The source code of AODV in $n s-2$ is modified to implement the proposed scheme. Table II lists the parameters used in the simulation. The key performance measures are end-to-end delay, packet loss ratio, routing overhead, route discovery frequency and energy consumption. Network topology is same as given in Section III. The obtained results are grouped into three sections. Section VI-A discuss the performance of the proposed scheme with respect to increasing number of connections in the network, the performance of the network is considered using increasing packet generation rate in Section VI-B. In Section VI-C network lifetime with respect to increase in number of nodes is compared. The simulation parameters for three scenarios mentioned are shown in Table III.

\section{A. Increasing number of connections}

The number of connections are increased from source to sink to evaluate the performance of the proposed scheme. To generate congestion in this scenario, packet transfer rate is assigned 200kbps. As shown in Fig. 2a, $C L-R S$ outperforms the layered AODV as the number of connections increase. $C L-R S$ shows lower packet loss ratio as it uses the hopto-hop reliable transmission ratio. AODV has high packet loss 


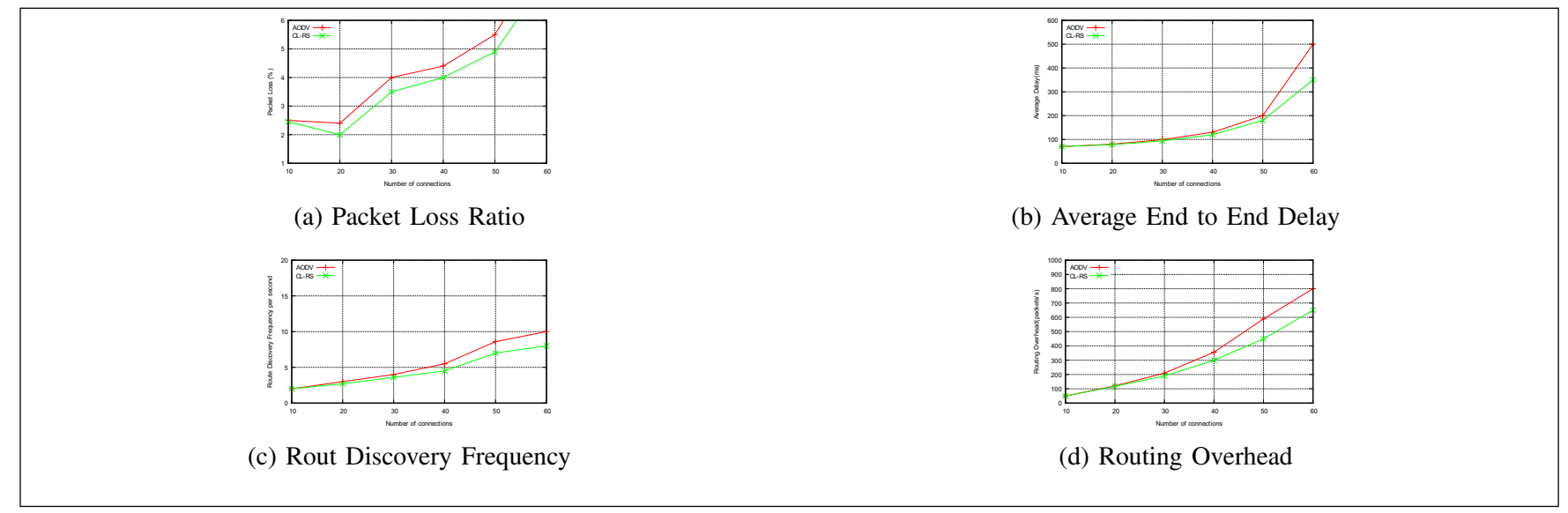

Fig. 2: Increasing number of connections

ratio as it does not test hop-to-hop link quality. In Fig. 2b, $C L-R S$ shows low end to end delay compared to AODV because it can effectively handle load of the node during route discovery process. In Fig. 2c, the average route discovery frequency of $C L-R S$ and AODV is calculated. $C L-R S$ uses low route discovery frequency. In Fig. 2 d, $C L-R S$ incurs low overhead as compared to AODV as it restricts the control packets only in its request zone.

\section{B. Varying Packet Generation Rate}

Rate of packet generation in the network is elevated to observe the effect of load in the network with regard to performance of the proposed scheme. As shown in Fig. 3a, the average packet loss ratio of $C L-R S$ is low compared to layered AODV. $C L-R S$ is capable of handling better delay. As shown in Fig. 3b, $C L-R S$ outperforms AODV because, AODV has no mechanism to reduce congestion on high traffic loads. $C L-R S$ shows low end to end because it considers current load of the network during hop to hop route discovery phase. Fig. 3c and Fig. 3d shows that $C L-R S$ outperforms AODV in terms of number of route discovery frequency and routing overheads.

\section{Increasing number of nodes}

The effect of increasing number of nodes has been observed on the performance of the proposed scheme. As shown in Fig. 4a, $C L-R S$ has low packet loss ratio compared to AODV because $C L-R S$ uses a local strategy of link quality estimation for route maintenance. Fig. $4 \mathrm{~b}$ shows that $C L-R S$ has lower delay compared to AODV because route paths are used only in request zone of the source node. With increasing number of nodes $C L-R S$ has lower routing overhead and route discovery frequency rate as shown in Fig. 4c and Fig. 4d. In Fig. 5, the average energy consumption in the network with varying network size is shown. It is observed that, $C L-R S$ has a low energy consumption compared to AODV. This is due to considering the residual energy during the route selection. Hence, the proposed cross layer joint routing scheme increases the network lifetime with minimum energy consumption.

\section{CONCLUSION}

This paper aims to prolong the network lifetime with reliability constraint using a cross layer joint routing scheme

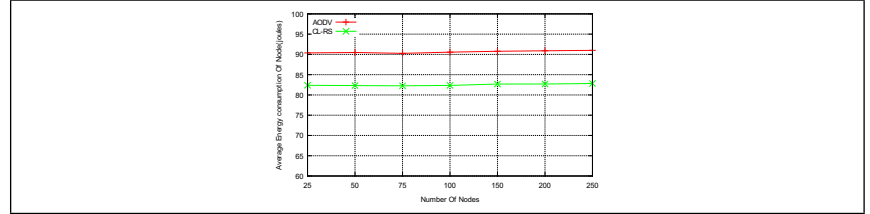

Fig. 5: Average energy

$(C L-R S)$ and cross layer MAC $(C L-M A C)$. The proposed scheme considers network layer and MAC layer of the protocol stack. This scheme balances the energy in the network by defining a joint cost function that enables routing decision. The simulation results are taken for different network sizes, different connections and different rate of transmission to measure the reliability and energy consumption. The performance of the proposed scheme is compared with layered AODV. The results demonstrates that the proposed scheme achieves high reliability with low energy consumption thereby increasing network lifetime. Devising analytical models of the proposed scheme and comparing with other cross layer algorithms in WSN is a part of our future work.

\section{REFERENCES}

[1] A. Leshem K.Cohen. A time-varying opportunistic approach to lifetime maximization of wireless sensor networks. IEEE Transactions on Signal Processing, 58(10):5307-5319, Oct 2010.

[2] M. Motani V. Srivastava. Cross-layer desing: a survey and the road ahead. IEEE Communications Magazine, 43(12):112-119, Dec 2005.

[3] Frank Aune. Cross-layer desing tutorial. Creative commons license, Nov 2004.

[4] C.J.Bleakleyy Kunjan Patel, Lim Jong Chery and Wim Vanderbauwhedez. Maw: A reliable lightweight multi-hop wireless sensor network routing protocol. International Conference on Computational Science and Engineering, 38(4):487-493, Nov 2009.

[5] Seira Ann George Monica R Mundada, Saavan Kiran and Raja Nahusha Varsha. A survey on energy efficient protocols for wireless sensor networks. Internatinal Journal of Computer Applications, 11(10):3540, Dec 2010.

[6] B. Shanthi B. Baranidharan. A study on energy efficient routing protocols in wireless sensor netowrks. International Journal of Distributed and Parallel Systems), 3(3):311-330, May 2012.

[7] Binbin Hao Changle Li, Hanxiao Zhang and Jiandong Li. A survey on routing protocols for large scale wireless sensor networks. Sensors, 11(4):3498-3526, Mar 2011 


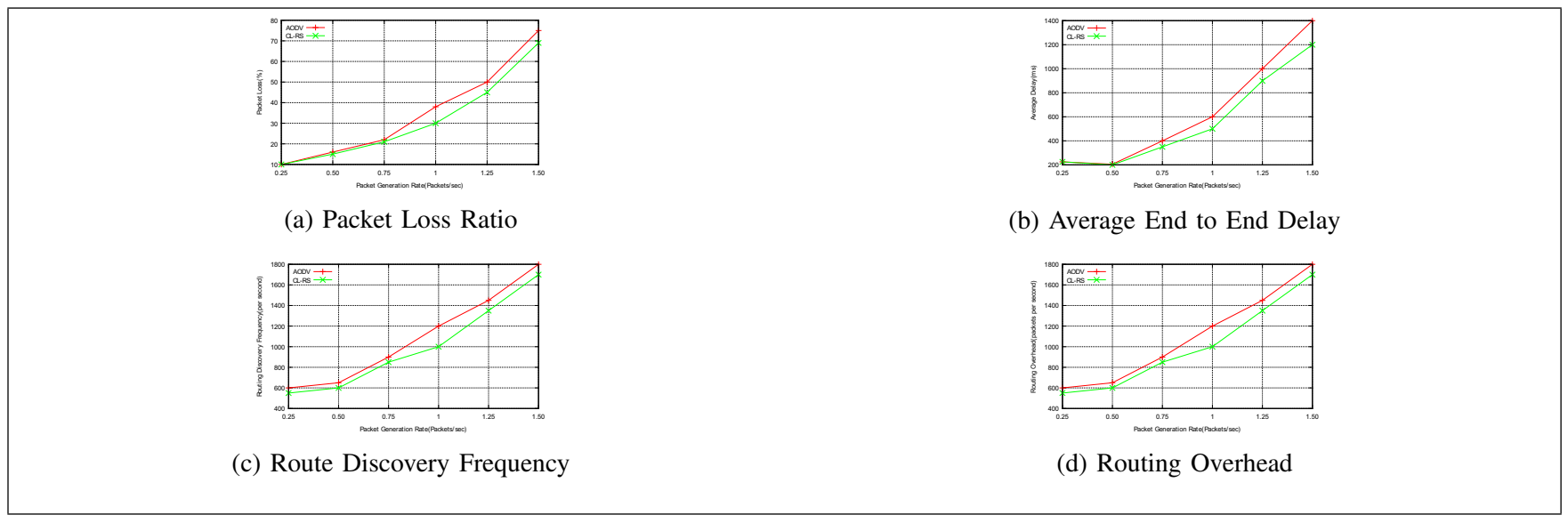

Fig. 3: Varying packets generation rate

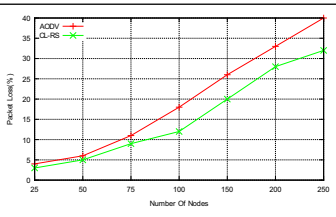

(a) Packet Loss Ratio

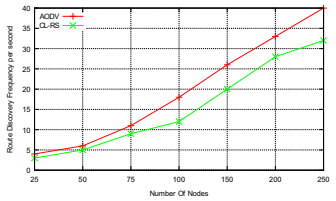

(c) Route Discovery Frequency

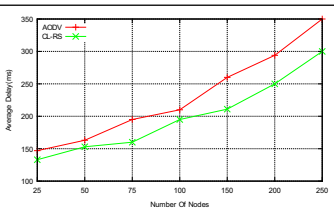

(b) Average End to End Delay

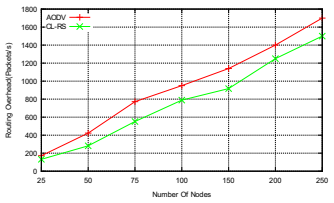

(d) Routing Overhead

Fig. 4: Increasing number of nodes

[8] Mehmet Yunus Donmez Sinam Isik and Cem Ersoy. Cross layer load balanced forwarding schemes for video sensor networks. Elsevier Ad Hoc Networks, 9(3):265-284, July 2010.

[9] Eduardo Cerqueira Kassio Machado, Denis Rosario and Jose Neuman de Souza. A routing protocol based on energy and link quality for internet of things applications. Sensors, 13(2):1942-1964, Feb 2013.

[10] Xiao Fu Zhenhua Yu and Mehmet C.Vuran. A reliable energy-efficient multi-level routing algorithm for wireless sensor networks using fuzzy petri nets. Sensors, 11(3):3381-3400, March 2011.

[11] Sabrina Sicari Alberto Coen-Porisini. Improving data quality using a cross layer protocol in wireless sensor networks. Elsevier Computer Networks, 56(17):3655-3665, August 2012.

[12] Ashfaq Khokhar Mohamed S. Hefeida, Turkmen Canli. Cl-mac: A cross-layer mac protocol for heterogeneous wireless sensor networks. Elsevier Ad Hoc Networks, 11:213-225, May 2012.

[13] Choong Seon Hong Mohammad Helal Uddin Ahmed, Md. Abdur Razzaque. Dec-mac: Delay and energy aware cooperative medius access control protocol for wireless sensor networks. Springer Annals Telecommunications, 68:485-501, Oct 2012.

[14] Guoliang Xing Jianping Wang, Deying Li and Hongwei Du. Cross-layer sleep scheduling design in service-oriented wireless sensor networks. IEEE Transactions on Mobile Computing, 9(11):1622-1633, Nov 2010.

[15] NS-2. The ns manual (formally known as ns documentaion). In http: //www.isi.edu/nsnam/ns/doc. 